Memoirs of the Faculty ofScience, Kyūsyū University

Ser. A, Vol. 7, No. 1, 1952

\title{
SUCCESSIVE PROCESSES OF STATISTICAL CONTROLS, $(1)^{1)}$
}

\author{
By \\ Tosio KITAgawa
}

(Received July 31, 1952)

\begin{abstract}
Introduction
Part I. Sequential processes of statistical controls.

$\S 1$. Formulation of sequential processes of statistical controls.

Part II. Successive processes of regulations to attain prescribed level of control.

$\S 1$. Introductory.

$\S 2$. Successive process of regulation without tests of significances.

$\S 3$. Successive process of regulation with testings significances.

$\S 4$. Cost considerations on successive process of regulation with testing of significance.
\end{abstract}

Contents

Successive processes of statistical controls,

\section{Introduction}

Statistical techniques adopted in quality controls such as control-chart methods due to SHEwHART [1] and inspection controls plans due to FRY [1], DODGE [1] and others have been developed by various authors beyond the scopes of classical theories of statistical inferences. In our previous papers [1] [4] we have emphasised the important features of our inductive behaviours which should appeal to successive processes of statistical inferences. Such inductive behaviours have been involved in the present practices of statistical inferences used in various applications of statistical theories. Statistical controls of quality of manufactured products belong to the most important applications. Neverthless it seems to the author of the present paper that any adequate theoretical foundation has not yet completely established, and also that such a foundation can be established only after a new reconstruction of modern mathematical statistics.

1) Part I and $\S 1, \S 3$ and $\S 4$ of Part II were communicated by the author at the Autumn Branch Meeting of Math. Soc. of Japan held at Kyoto Univ., in October, 201951 , while $\$ 2$ of Part II in its present general formulation was enunciated at the Annual Meeting of the same Soc. held at Tokyo Univ., in June 6, 1952. 
Our points of view in planning a new formulation of statistical inferences and controls may be perhaps said to be beyond the present realm of mathematical statistics, and our general formulations were discussed in our previous paper [5].

In the present paper, we shall be contented with restricting ourselves within more technical problems chiefly associated with automatic controls of pulsed data or statistical time series.

Even in such restricted formulations of our problems it may be still worth while to notice that there are three main items on which we should reckon in planning and evaluating the job of statistical controls, i.e., $\left(1^{\circ}\right)$ objects of controls, $\left(2^{\circ}\right)$ means (methods) of controls and $\left(3^{\circ}\right)$ costs of controls. It will not be useless to explain the meanings of these three items :

$\left(1^{\circ}\right)$ The objects of controls. There are various types of goals to which our statistical controls will aim to reach. They may be however classified into two broad types of which (a) the first type is consisted of those statistical controls which aim to attain to certain prescribed levels of controls from given levels, and (b) the second consisted of those statistical controls which aim to maintain certain levels of controls already attained in our given states.

$\left(2^{\circ}\right)$ The means of statistical controls. In our terminology these concern themselves with inductive behaviours and not with the physical apparata which secure us to attain our object of controls mechanically or electrically. In the present practices of statistical inferences adopted in quality-controls we appeal to one of the following procedures.

(a) Draw samples from batches or from its supply, and make estimations about batches or its supply. According to these estimations we shall make certain types of controls.

(b) Draw samples from batches or from its supply, make certain statistics by means of which we may be able to make testing hypotheses. According to the significances or non-significances of these tests, let us make certain types of controls and no controls respectively.

$\left(3^{\circ}\right)$ The costs of controls. The cost considerations have been mainly developed on sampling survey techniques, and it does not seem to us to be fully nor throughly discussed on the literatures of quality controls from the theoretical standpoints in spite of their real importances in engineerings.

If any costs of sampling should be taken into our consideration, it will be non the less important to reckon the costs of controls. Our probabilistic approaches to theories of statistical controls may be distinguished from 
those of statistical inferences in the sense that the former theories concerns themselves with inferences and actions, while the latter merely with inferences. The following three assertions may be recongnised as serving in making clear fundamental characteristic features which should be more or less enunciated and formulated in any useful theory of statistical controls :

(1 ${ }^{\circ}$ To make our inference with more confidence or with more accuracy means in general to allocate more costs to our inductive behaviours, and hence it will reduce costs to be spent upon action.

$\left(2^{\circ}\right)$ On the contrary, in order to reserve more active resources for our action, let us be contented with less knowledge and hence with poorer inferences. Then our situations will be such that we possess more active resources reserved for action and neverthless that our purposes of actions may be rather inaccurate or altogather ill-designed.

$\left(3^{\circ}\right)$ Inaccurate inferences may involve us into nervous vibrating situations of fluactuating sequences of actions.

The other characteristic features of our probabilistic approches lie in the formulation that the interrelations and communications between measuring means (inductive behaviours and communications) and controlling means (actions) are defined schematically by some rules. In this point of view our present approaches may be rather called as cybernetical theory of statistical controls in the sense of WIENER [1]. Neverthless it may be of some use in underatanding and evaluating the theoretical aspects of statistical controls of quality, which are not generally automatic controls and which appeal to the skills and powers of technical judgements of engineers.

Further charateristic feature of our approaches lie in formulating successive processes in our controlling behaviours. In our previous papers $[1] \sim[4]$ we have emphasised successive processes in statistical inferences, and similar considerations of successive processes seem to us to be indispensable for controlling behaviours.

Indeed the idea of successive processes of statistical controls will be seen to be deeply associated with our actions such as (1) process of attainments to an objects, (2) regulating process, (3) searching process, (4) following up process, (5) stragetic process and (6) process of preventions and protections.

In Part I of this first paper of ours we shall treat the formulation of sequential processes of statistical controls, and we shall devote Part II to probabilistic approaches to some fundamental controlling behaviours. 


\section{Part I. Sequential processes of statistical controls}

\section{§ 1. Formulation of sequential processes of statistical controls.}

A general formulation of successive processes of statistical controls, which will correspond to that of successive processes of statistical controls enunciated in Part VIII in our previous paper [3], will not be discussed here, and we shall restrict ourselves with a certain special one of such formulations, which. may be recognised to correspond to the statistical decision function due to WALD [1]. Owing to the reasons illustrated in Part VIII in our previous paper [3], we shall call these processes as sequential process of statistical controls.

In short what we aim to formulate are concerned with the following two: $\left(1^{\circ}\right)$ to recognise that our available powers should be suitably allocated to both of inferences and actions, and $\left(2^{\circ}\right)$ to attain our aims in sequential processes of inferences and actions.

Let us adopt the definitions and terminologies of WALD [1] introduced in his theory of statistical decision function as far as possible. In stead of definining statistical decision functions, however, what we should define after all is that which may be called statistical control-decision function $\gamma$. By experimentation $d^{e}$ we mean making observations $x=\left\{x_{i}\right\}$ on some of the chance variables in the sequence $X=\left\{X_{i}\right\}$, and let $D^{e}$ be the space of all $d^{e}$ which may be said to be a finite (non-empty) subset of the set $I$. of all positive integers. By a controlling action $c$ we mean a transformation of the stochastic process $X=\left\{X_{i}\right\}$ into another stochastic process $X^{c}=\left\{X_{i}^{c}\right\}$. For example let $\left\{X_{i}\right\}$ be a sequence of independent stochastic variables each of which is distributed in a normal distribution $N\left(\xi_{i}, \sigma_{i}^{2}\right)$. Let controlling action $c$ be a shifting to each stochastic variable by the amount $a$. Then $x_{i}{ }^{c}$ becomes $X_{i}+a$ which is distributed according to $N\left(\xi_{i}+a, \sigma_{i}{ }^{2}\right)$. Let $D^{c}$ be the space of all possible manners of controlling actions $d^{c}$ adopted in our formulation.

For the sake of generality and brevity we shall assume that to perform no actions on our object will be regarded as a control belonging to $D^{c}$. Let us denote by $d^{t}$ and $D^{t}$ a terminal decision and the space of all possible terminal decisions $d^{t}$. The spaces $D^{c}$ and $D^{t}$ are to be regarded as data of the control problem and will vary with the problem under consideration. Further let $D_{i_{1}}{ }^{e}, i_{2}, \cdots, i_{k}$ be the space of all elements $d^{e}$ of $D^{e}$ which are subsets of the set $I-\left\{i_{1}, i_{2}, \cdots, i_{k}\right\}$, and let $D_{i_{1}}{ }^{c}, i_{2}, \ldots, i_{k}$ be the space of all possible controlling actions $d^{c}$ which are to be applied on every stochastic variable $X_{j}$ so long as $j$ belongs to the set $I-\left\{i_{1}, i_{2}, \cdots, i_{k}\right\}$. 
By a paired set of experimentation after control, which will be denoted by $d^{c e}$ or by $\left(d^{c}, d^{e}\right)$, we mean that we make a controlling action $d^{c}$ on the stochastic process and then make experimentation $d^{e}$ on new stochastic processes transformed by $d$.

Let us denote by $D_{i_{1}}{ }^{c e}, i_{2}, \ldots, i_{k}$ the set of all paired sets of experimentations after controls $d^{e e}=\left(d^{c}, d^{e}\right)$ where $d^{c}$ and $d^{e}$ belong to the sets $D_{i_{1}}{ }^{c}, i_{2}, \ldots, i_{k}$ and $D_{i_{1}}{ }^{e}, i_{2}, \ldots, i_{k}$ respectively.

Let $D_{i_{1}, i_{2}, \ldots, i_{k}}$ be the set-theoretical sum of $D_{i_{1}}{ }_{1}, i_{2}, \ldots, i_{k}$ and $D^{t}$ : i.e., it consists of all elements $\left(d^{c}, d^{e}\right)$ of $D_{i 1}{ }^{e}, i_{2}, \ldots, i_{k}$ and all elements $d^{t}$ of $D^{t}$, and let $D$ be the set-theoretical sum of $D^{c e}$ and $D^{t}$.

After these preparations we shall introduce the following definition. A function $d\left(x ; c_{1}, s_{1}, c_{2}, s_{2}, \ldots, c_{k}, s_{k}\right)$ is said to be a nonrandomized controldecision function if the following four conditions are fulfilled:

$\left(1^{\circ}\right)$ It is a single-valued function defined for all positive integral values $k$, for any sample point $x$, for any finite disjoint sets $s_{1}, s_{2}, \cdots, s_{k}$ and for any set of controlling actions $c_{1}, c_{2}, \cdots, c_{k}$.

$\left(2^{\circ}\right)$ The value of $d\left(x ; c_{1}, s_{1}, \ldots, c_{k}, s_{k}\right)$ is independent of the coordinates $x_{i}$ of $x$ for which the integer $i$ is not contained in any of the sets $s_{1}, s_{2}, \cdots, s_{k}$.

$\left(3^{\circ}\right)$ For $k \geqq 1$, the value of the function $d\left(x ; c_{1}, s_{1}, \cdots, c_{k}, s_{k}\right)$ may be any element of $D_{i_{1}, i_{2}, \ldots, i_{r}}$, where $\left\{i_{1}, i_{2}, \cdots, i_{r}\right\}$ is the set-theoretical sum of $s_{i}, s_{2}, \cdots, s_{k}$.

$\left(4^{\circ}\right)$ It is a constant when $k=0$, which we denote by $d(O) . d(O)$ may be any element of $D^{c e} \cup D^{t}$.

Such a system of control-decision functions can be used to determine uniquely a rule for carrying out the experimentation and the controlling actions and for selecting an element $d^{t}$ of $D^{t}$. In fact our procedure can be done in the following manner:

$\left(1^{\circ}\right)_{1}$ If $d(O)$ is an element $d^{t}$ of $D^{t}$, no experimentation and no controlling action are performed, and the terminal decision $d(O)$ is chosen.

$\left(1^{\circ}\right)_{2}$ If $d(O)$ is an element $\left(d_{1}{ }^{c}, d_{1}^{e}\right)$ with $d_{1}^{e}=s_{1}=\left(i_{1}, i_{2}, \cdots, i_{r}\right)$ of $D^{e}$, then the controlling action $d_{i}{ }^{c}=c_{1}$ is performed on all stochastic variables $X_{j}$, and the observations are made on the transformed stochastic variables $X_{i_{1}}{ }^{c}, X_{i_{2}}{ }^{c}, X_{i_{3}}{ }^{c}, \cdots, X_{i r}{ }^{c}$, and the value $d\left(x ; c_{1}, s_{1}\right)$ is calculated.

$\left(2^{\nu}\right)_{1}$ If $\boldsymbol{d}\left(x ; c_{1}, s_{1}\right)$ is an element $\boldsymbol{d}^{t}$ of $D^{t}$, experimentations and controlling actions are stopped with the terminal decision $d\left(x ; c_{1}, s_{1}\right)$. 
$\left(2^{\circ}\right)_{2}$ If $d\left(x ; c_{1}, s_{1}\right)$ is an element $\left(d_{2}^{c}, d_{2}^{e}\right)$ with $d_{2}^{e}=s_{2}=\left(j_{1}, j_{2}, \cdots\right.$, $j_{u}$ ), then the controlling action $d_{2}{ }^{c}=c_{2}$ is performed on every stochastic variable $X_{j}$ so long as $j$ belongs to the set $I-\left\{i_{1}, i_{2}, \cdots, i_{r}\right\}$, and the

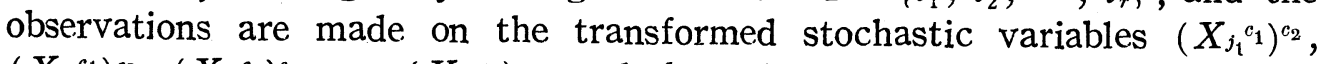
$\left(X_{j_{2}}{ }^{{ }_{1}}\right)^{c_{2}},\left(X_{j 3}{ }^{{ }{ }^{1}}\right)^{c_{2}}, \cdots,\left(X_{j_{u}}{ }^{c_{1}}\right)^{c_{2}}$, and the value $d\left(x ; c_{1}, s_{1}, c_{2}, s_{2}\right)$ is calculated.

These sequential procedures are furthermore applied on $d\left(x ; c_{1}, s_{1}, c_{2}, s_{2}\right)$ and so on. For the sake of simplicity, we may and we shall write $\left(X_{k}^{c_{1}}\right)^{c_{2}}$ by $X_{k}^{c_{1} c_{2} 2}$, and similarly for $X_{k}^{c_{1} c_{2} c_{3}}$ and so on.

Let us now define randomized control-decision functions. Let $C^{D}$ be a certain Borel field of subsets of the space $D$, which contains all the denumerable subsets of $D^{c e}$. By a probability density measure $o$ on the space $D$ we shall mean a probability measure defined for all elements of the Borel field $C_{\nu}$. Let $\Delta$ be the space of all probability measures $\delta$. For any subset $\left\{i_{1}, i_{2}, \cdots, i_{k}\right\}$ of $I$, let $\Delta\left(i_{1}, i_{2}, \cdots, i_{k}\right)$ be the class of all probability measures $\delta$ for which $o\left(D_{i_{1}}, i_{2}, \ldots, i_{k}\right)=1$.

A function $\delta\left(x ; c_{1}, s_{1}, \cdots, c_{k}, s_{k}\right)$ whose values are elements of $J$ is said to be a randomized decision function if $:\left(1^{\circ}\right)$ it is a single-valued function defined for any positive integer $k$, for any finite disjoint sets $s_{1}, s_{2}, \ldots, s_{k}$, for any set of $c_{1}, c_{2}, \cdots, c_{k}$, and for any sample point $x ;\left(2^{\circ}\right) \delta\left(x ; c_{1}, s_{1}\right.$, $\left.\cdots, c_{k}, s_{k}\right)$ is an element of $\Delta\left(i_{1}, i_{2}, \cdots, i_{r}\right)$ if $r \geqq 1 ;\left(3^{\circ}\right)$ the value of $o\left(x ; c_{1}, s_{1}, \cdots, c_{k}, s_{k}\right)$ is independent of the coordinates $x_{i}$ of $x$ for which the integer $i$ is not contained in any of the sets $s_{1}, s_{2}, \ldots, s_{k} .\left(4^{\circ}\right)$ it is a constant $\delta(O)$ belonging to $\Delta$ when $k=0$.

It is to be noticed here that in our formulation sample $x$ should be distinguished from our real observation in experimentation after controls.

Let us put $s_{j}=\left(i_{j_{1}}, i_{j_{2}}, \cdots, i_{j n}\right) x_{s_{j}}=\left(x_{i_{j 1}}, x_{i_{2}}, \cdots, x_{i_{n n}}\right)$ and $x_{s_{j_{1}}{ }^{c}}$ $=\left(x_{i_{j 1}}{ }^{c}, x_{i_{j 2}}{ }^{c}, \cdots, x_{i_{j n}{ }^{c}}\right)$. Then our real observation will be denoted by

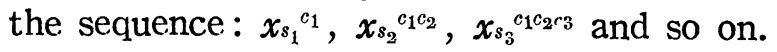

Now let us denote the cost of experimentations by $c\left(x ; s_{1}, s_{2}, \cdots, s_{k}\right)$ when the experiment was carried out in $k$ stages according to the manner which WALD [1] has introduced, and furthermore the costs of controlling actions by $c\left(x ; c_{1}, c_{2}, \cdots, c_{k}\right)$ in the similar manner when the successive actions of controls were $d_{1}{ }^{c}=c_{1}, d_{2}{ }^{b}=c_{2}, \cdots, d_{k-1}^{c}=c_{k-1}$ and $d_{k}^{c}=c_{k}$. We shall also use $\delta(x ; c s)$ as an alternative notation for $\delta\left(x ; c_{1}, s_{1}, c_{2}, s_{2}\right.$, $\left.\cdots, c_{k}, s_{k}\right), c\left(x ; d_{12} \cdot k^{e}\right)$ for $c\left(x ; s_{1}, s_{2}, \cdots, s_{k}\right)$ and $c\left(x ; d_{12} k^{e}\right)$ for $c\left(x ; c_{1}, c_{2}, \cdots, c_{k}\right)$.

Let us denote by $F(x)$ the probability distribution of the stochastic 
variable $X$, that is, $F(x)=\operatorname{Pr} .\left\{X_{i}<x_{i},(i=1,2,3, \cdots)\right\}$ when $x=\left(x_{1}\right.$, $\left.x_{2}, \cdots, x_{n}, \cdots\right)$. It is one of the characteriatic features of our formulation to take into consideration the transformations of stochastic processes. We shall hence denote by $F_{c}(x)$ the probability. distribution of $X^{c}$, by $F_{c_{1} c_{2}}(x)$ that of $X^{c_{1} c_{2}}$ and so on. Let $M$ be the whole sample space.

If the r. d. c. f. $\delta(x ; s c)$ is adopted and if $x=\left\{x_{i}\right\}$ is the sample point, then the probability that the experiment after control will be carried out in $k$ stages, the first stage in accordance with $d_{1}^{c e}$, the second in accordance with $\boldsymbol{d}_{2}^{c e}, \cdots$, the $k$-th stage with accordance with $\boldsymbol{d}_{k}{ }^{c e}$, and that the terminal decision will be an element of the subset $\bar{D}^{t}$ of $D^{t}$ is given by

$$
\begin{aligned}
& p\left(d_{1}^{c e}, d_{2}^{c e}, \cdots, d_{k}^{c e}, D^{t} / x, \delta\right) \\
& =\delta\left(d_{1}^{c e} / O\right) \delta\left(d_{2}^{c e} / x^{c_{1}} ; d_{1}^{c e}\right) \delta\left(d_{3}^{c e} / x^{c c^{c}} ; d_{1}^{r e}, d_{2}{ }^{c e}\right) \\
& \delta\left(\boldsymbol{d}_{k}^{c e} / \boldsymbol{x}^{c_{1} c_{2} \cdots c_{k-1}} ; \boldsymbol{d}_{1}^{c e}, \boldsymbol{d}_{2}^{c e}, \cdots, \boldsymbol{d}_{k-1}^{c e}\right) \\
& \grave{o}\left(D^{t} / X^{c_{1} c_{2} \cdots c_{k}} ; d_{1}^{c e}, d_{2}^{r e}, \cdots, d_{k}^{{ }^{c e}}\right) \text {. }
\end{aligned}
$$

For $k=0$, the right-hand member of $(1.01)$ reduces to $\delta\left(\bar{D}^{\prime} / O\right)$. For the sake of simplicity, we shall denote $(10.1)$ by $p\left(d_{2 \ldots k}{ }^{c_{e}}, \bar{D}^{t} / x, \delta\right)$.

To each assigned set $s=\left(s_{1}, s_{2}, \cdots, s_{k}\right)$ the set of all $\left(d^{c_{1 e_{1}}}, d^{c_{2 e_{2}}}, \cdots\right.$, $\left.d^{c_{k} k^{\prime} k}\right)$ will be denoted by $D^{c_{1} c_{2} \ldots c_{k}}(s)$, and in what follows we assume that a certain probability dense measure $\gamma_{s}(c)$ is defined for a certain Borel set $\Gamma_{c}(s)$ of the subsets of $D^{c_{1} c_{2} \cdots c_{k}}(s)$.

The expected value of the cost of controlling actions when $F$ is true and the control-decision function $\delta(x ; c s)$ is adopted is given by

(1.02) $r_{c}(F, \delta)$

$$
=\sum_{k=1}^{\infty} \sum_{e_{1} \cdots \epsilon_{k}} \int_{\Gamma_{c}(s)} \int_{M} p\left(d_{12 \cdots k}{ }^{c e}, D^{t} / x, \delta\right) c\left(x ; d_{12 \cdots k}^{o}\right) d \gamma_{s}(c) d F_{c_{1} \cdots c_{k}}(x) .
$$

The expected value of the cost of experimentation when $F$ is true and the control-decision function $\delta(x ; c s)$ is adopted is given by

$$
\begin{aligned}
& r_{e}(F, \delta) \\
= & \sum_{k=1}^{\infty} \sum_{e_{1} \cdots e_{k}} \int_{\Gamma_{e}(s)} \int_{M} p\left(d_{12 \ldots k} c_{e}^{e}, D^{t} / x, \delta\right) c\left(x ; d_{12 \ldots k^{e}}\right) d \gamma_{s}(c) d F_{c_{1} \cdots c_{k}}(x)
\end{aligned}
$$

Let us denote by a non-negative funnction $W\left(F, d^{t}\right)$, defined any pair $\left(F, d^{t}\right), F \in \Omega$ and $d^{t} \in D^{t}$, the suffer by making the terminal decision $d_{t}$ when $F$ is the true distribution. 
The excepted value of the loss $W\left(F, d^{t}\right)$ when our control-decision function $\delta$ is used and $F$ is true is equal to

$$
\begin{aligned}
& \boldsymbol{r}_{d}(F, \delta) \\
= & \sum_{k=0}^{\infty} \sum_{e_{1}} \sum_{\ell_{k}} \int_{\Gamma_{c}(s)} W\left(F_{c_{1} c_{2} \cdots c_{k}}, d^{t}\right) d P\left(d_{12 \cdots k}, \bar{D}^{t} / F, \delta\right),
\end{aligned}
$$

where we have put

$$
\begin{aligned}
& P\left(d_{12 \cdots k} c_{e}, D^{\bar{t}} / F, \grave{o}\right) \\
= & \int_{M} p\left(d_{12 \cdots k}{ }^{c e}, \bar{D}^{t} / x, \grave{o}\right) d F_{c_{1} c_{2} \cdots c_{k}}(x) .
\end{aligned}
$$

After these preparation the $\operatorname{risk} r(F, \delta)$ is defined as the sum :

$$
r(F, \grave{o})=r_{e}(F, \grave{o})+r_{c}(F, \grave{o})+r_{d}(F, \grave{o}) .
$$

If an a priori distribution $\xi$ on $Q$ exists and is known to the control man, the average risk may be introduced:

$$
r^{*}(\xi, \delta)=\int_{\Omega} r(F, \delta) d \xi .
$$

We reach a position to ennunciate that our whole formulation thus developed takes analogous form as to that of WALD in the statistical decision functions. We shall not enter here detailed discussions on our formulation to establish various theorems concerning our control-decision functions. One of the real significances of our formulations different from WALD's formulation lies in the transformations of $F$.

\section{Part II. Successive processes of regulations to attain prescribed level of control}

$\S 1$. Introductory. In this Part we shall discuss the following fundamental problem, which will serve as a guide to lead us to a general formulation of successive processes of regulations:

Problem A. Let us assume that in a given sequence (stochastic process) $X=\left\{X_{i}\right\}$ of mutually independent stochastic variables $X_{i}$ the probability distribution function of each $X_{i}$ is given by a normal distribution $N\left(m_{i}, \sigma^{2}\right)$ $(i=1,2, \cdots$,$) with unknown population means m_{i}$, while the common variance $\sigma^{2}$ is known to us. What we want to attain is to obtain a normal distribution $N\left(\xi, \sigma^{2}\right)$, where $\xi$ is a prescribed value for population mean. 
There are two fundamental types of successive control-decision procedures. Any successive control-decision procedure may be classified into one of these two types whether the procedure is provided with testing of significances or not. In $\S 2$ we shall discuss the case when no testing of significances are performed and our statistical inferences mean some estimations about the mean values of populations, and in $\S 3$ certain of its generalisations. In $\S 4$ we turn to the case when testing of significances are used in each step of procedures.

\section{\$2. Successive process of regulation without tests of significances.}

Under the general hypothesis to Problem A, let us consider the following successive process of statistical inferences and controls:

$\left(1^{\circ}\right)$ Let $O_{n_{1}}$ be a random sample of size $n_{1}$ drawn from the normal population $N\left(m_{1}, \sigma^{2}\right)$, and let $\bar{x}_{1}$ be the sample mean defined for $O_{n_{1}}$.

Let us perform an action $c_{1}$ which means the shift of the population means $m_{i}$ into

$$
m_{i}{ }^{c}=m_{i}+f_{1}\left(\xi-\bar{x}_{1}\right), \quad(i=2,3,4, \cdots) .
$$

$\left(2^{\circ}\right)$ Let $\mathrm{O}_{n_{2}}$ be a random sample drawn of size $n_{2}$ from the normal population $N\left(m_{2}+f_{1}\left(\xi-\bar{x}_{1}\right), \sigma^{2}\right)$, and let $\bar{x}_{2}$ be the sample mean defined for $\mathrm{O}_{n_{2}}$.

Let us perform an action $c_{2}$ which means the shift of population means $m_{i}^{c_{1}}$ into $\left(m_{i}^{c_{1}}\right)^{c_{2}}=m_{i}{ }^{{ }^{1} c_{2}}$ for $i=3,4,5,6, \cdots$,

$$
m_{i}^{c_{1} c_{2}}=m_{i}^{c_{1}}+f_{2}\left(\xi-\bar{x}_{2}\right)=m_{i}+f_{1}\left(\xi-\bar{x}_{1}\right)+f_{2}\left(\xi-\bar{x}_{2}\right) .
$$

$\left(3^{\circ}\right) \sim\left(k^{\circ}\right)$ We shall proceed similarly to draw a sample of size $O_{n_{3}}$ and to perform an action $c_{3}$ which is defined by $m^{c_{1} c_{2} c_{3}}=m^{c_{1} c_{2}}+f_{3}\left(\xi-\bar{x}_{3}\right)$, and so on. These proceedures will be continued until the $k$-th step which will define for $j \geq k+1$

$$
\begin{aligned}
m_{j}^{c_{1} c_{2} \cdots c_{k}} & =m_{j}^{c_{1} c_{2} \cdots c_{k-1}}+f_{k}\left(\xi-\bar{x}_{k}\right) \\
& =m_{j}+\sum_{j=1}^{k} f_{j}\left(\xi-x_{j}\right) .
\end{aligned}
$$

This is an extremely simplified model to our control-decision processes. Neverthless it will serve us to make clear some characterislic features of statistical control problems.

The sample sizes $\left\{\boldsymbol{n}_{i}\right\}(i=1,2,3, \cdots)$ are assumed to be assigned a priori to our processes. This assumption is also a simplication which will serve 
to facilitate our mathematical analysis without introducing us really sequential processes.

Furthermore we shall restrict ourselves with the following special case when each $f_{i}$ becomes a linear homogenous function of $\xi-\bar{x}_{i}$ with prescribed constants $c_{i}$, i.e.,

$$
f_{i}\left(\xi-\bar{x}_{i}\right)=c_{i}\left(\xi-\bar{x}_{i}\right), \quad(i=1,2, \cdots, k) .
$$

We shall observe

Theorem 2.1. Under our hypothesis (2.04) concerning the successive control procedure defined in this paragraph, after our control procedures, the sample means $\bar{x}_{j}$ defined for samples of size $n_{j}$ from the $j$-th parent population are distributed according to the normal distribution $N\left(m_{j}^{*}, \sigma_{j}^{* 2}\right)$ where for $j \geqq k+1$

$$
\begin{aligned}
m_{j}^{*} & =\left\{1-\prod_{i=1}^{k}\left(1-c_{i}\right)\right\} \xi \\
& +m_{j}-\sum_{i=1}^{k}\left\{\prod_{\nu=i+1}^{k}\left(1-c_{\nu}\right)\right\} c_{i} m_{i} \\
\sigma_{j}^{* 2} & =\sigma^{2}\left\{n_{j}{ }^{-1}+\sum_{i=1}^{k} n_{i}{ }^{-1} c_{i}{ }^{2} \prod_{\nu=i+1}^{k}\left(1-c_{\nu}\right)^{2}\right\}
\end{aligned}
$$

with $c_{k+1}=0$.

Before proceeding to the proof of our Theorem, it may be of some use to note the following special cases.

Example 2.1. Specially when all $m_{i}$ are equal to a constant $m$ independent of $i$ for all $i$, then, from (2.05), we have

$$
\begin{aligned}
m_{j}^{*} & =\left\{1-\prod_{i=1}^{k}\left(1-c_{i}\right)\right\} \xi+\prod_{i=1}^{k}\left(1-c_{i}\right) m . \\
& =\xi+(m-\xi) \prod_{i=1}^{k}\left(1-c_{i}\right) .
\end{aligned}
$$

This shows the effect of $k$ controls aiming to attain the standards $\xi$.

Example 2.2. Specially when there is an integer $l$ for which $c_{\nu}=1$, $(l \leqq \nu \leq k)$, then

$$
\begin{aligned}
& m_{j}^{*}=\xi+m_{j}-\sum_{i=l+1}^{k}\left\{\prod_{\nu=i+1}^{k}\left(1-c_{\nu}\right)\right\} c_{i} m_{i} . \\
& \sigma_{j}^{* 2}=\sigma^{2}\left\{n_{j}^{-1}+\sum_{i=l+1}^{k} n_{i}{ }^{-1} c_{i}{ }_{\nu=i+1}^{k}\left(1-c_{\nu}\right)^{2}\right\} .
\end{aligned}
$$


In the case when $l=k$, we have

$$
\begin{gathered}
m_{j}^{*}=\xi+m_{j}-m_{k} \\
\sigma_{j}^{* 2}=\sigma^{2}\left\{n_{j}^{-1}+n_{k}^{-1}\right\} .
\end{gathered}
$$

Thus $c_{l}=1$ may be said to keep an accurate count of all previous matters.

Example 2. 3. Specially when all $m_{i}$ coincide with a constant $m$, all $\boldsymbol{n}_{\boldsymbol{i}}$ with a constant $n$ and all $c_{i}$ with a constant $c$, then we have

$$
\begin{aligned}
& m_{j}^{*}=\xi+(m-\xi)(1-c)^{k} \\
& \sigma_{j}^{* 2}=\sigma^{2} n^{-1}\left[1+c^{2}\left(1-(1-c)^{2 k}\right)\left(1-(1-c)^{2}\right)^{-1}\right] .
\end{aligned}
$$

These show some aspects of accumulative effects introuced by a repetition of actions.

Proof of Theorem 2.1. For the sake of simplicity of notation let us consider the case when $\cdot j=k+1$. Then the probability density that we shall obtain $\left(\bar{x}_{1}, \bar{x}_{2}, \bar{x}_{3}, \cdots, \bar{x}_{k}, \bar{x}_{k+1}\right)$ can be readily seen from the equation. Let the events $E_{1}, E_{2}, \cdots, E_{k}$ and $E_{k+1}$ be defined as those which satisfy each of the following relations:

$$
\begin{aligned}
\text { (1) } & E_{1}: \bar{x}_{1}<X_{1}<\bar{x}_{1}+d \bar{x}_{1} \\
\text { ( } i) & E_{i}: \bar{x}_{i}<X_{i}^{{ }_{1} c_{2} \cdots c_{i-1}}<\bar{x}_{i}+d \bar{x}_{i}, \quad(i=2,3, \cdots, k) \\
(k+1) & E_{k+1}: \bar{x}_{k+1}<X_{k+1}{ }^{c_{1} c_{2} \cdots c_{k}}<\bar{x}_{k+1}+d \bar{x}_{k+1},
\end{aligned}
$$

where $d \bar{x}_{i}$ may be understood symbolically.

Then we have

$$
\begin{aligned}
& \operatorname{Pr} .\left\{E_{1} \cap E_{2} \cap \cdots \cap E_{k} \cap E_{k+1}\right\} \\
& \quad=\prod_{i=1}^{k+1}(2 \pi)^{-1 / 2} \sigma^{-1} n_{i}^{1 / 2} \exp \left[-n_{i}\left(2 \sigma^{2}\right)^{-1}\left(\overline{\boldsymbol{x}}_{i}-\boldsymbol{u}_{i-1}\right)^{2}\right] d \overline{\boldsymbol{x}}_{i},
\end{aligned}
$$

where for $i=2, \cdots, k+1$

$$
u_{i-1}=\bar{x}_{i}-\left(m_{i}+\sum_{\nu=1}^{i-1} c_{\nu}\left(\xi-\bar{x}_{\nu}\right)\right) \text {. }
$$

with $c_{k}=0$ and $u_{0}=0$. The first change of integration variables $\left(\bar{x}_{1}, \bar{x}_{2}\right.$, $\left.\cdots, \bar{x}_{k+1}\right)$ into $\left(u_{1}, u_{2}, \cdots, u_{k}, \bar{x}_{k+1}\right)$ and the second one of $\left(u_{1}, u_{2}, \cdots, u_{k}\right.$, $\left.\bar{x}_{k+1}\right)$ into $\left(v_{1}, v_{2}, \cdots, v_{k}, \bar{x}_{k+1}\right)$ which are defined

(2. 151) $\quad v_{1}=c_{1}^{-1} u_{1}$

$$
v_{i}=c_{i}^{-1} u_{i}-\left(c_{i}^{-1}-1\right) u_{i-1} \quad(i=2,3, \cdots, k)
$$

will give us 


$$
\begin{aligned}
& \operatorname{Pr}\left\{\left\{E_{1} \cap E_{2} \cap \cdots \cap E_{k} \cap E_{k+1}\right\}\right. \\
& \begin{array}{r}
\prod_{i=1}^{k}(2 \pi)^{-1 / 2} \sigma^{-1} n_{i}^{-1 / 2} \exp \left[-n_{i}\left(2 o^{2}\right)^{-1}\left(v_{i}-\xi-r_{i}\right)^{2}\right] d v_{i} \\
(2 \pi)^{-1 / 2} \sigma^{-1} n_{k+1}^{-1 / 2} \exp \left[-n_{k+1}\left(2 \sigma^{2}\right)^{-1}\left\{\bar{x}_{k+1}\right.\right. \\
\left.\left.-l\left(v_{1}, \cdots, v_{k}\right)\right\}^{2}\right] d \bar{x}_{k+1},
\end{array}
\end{aligned}
$$

where we have put

$$
\begin{aligned}
& \eta_{1}=\left(1-c_{1}^{-1}\right) m_{1}-\left(m_{2}-m_{1}\right) c_{1}^{-1} \\
& \eta_{i}=\left(m_{i+1}-m_{i}\right) c_{i}^{-1} \quad(i=2,3, \cdots, k) . \\
& l\left(v_{1}, v_{2}, \cdots, v_{k}\right)=\sum_{i=1}^{k}\left\{\prod_{\nu=i+1}^{k}\left(1-c_{\nu}\right)\right\} c_{i} v_{i} .
\end{aligned}
$$

The integration of the right-hand side of (2.16) over the whole $k$-dimensional Euclidian sample space $\left(v_{1}, v_{2}, \cdots, v_{k}\right)$ will lead us to the results to be proved.

§. Successive process of regulation with testings significances. Under the general hypothesis to Problem A, let us consider the following successive processes of statistical inferences and controls associated with tests of significances :

$\left(1^{\circ}\right)$ Let $O_{n_{1}}$ be a random sample of size $n_{1}$ drawn from the normal population $N\left(m_{1}, \sigma^{2}\right)$, let $x_{1}$ be the sample mean defined for $O_{n_{1}}$, and let us test the hypothesis $H_{1}: m_{1}=\xi$.

$\left(1^{\circ}\right)_{1}$ If $H_{1}$ becomes non-significant in $\left(1^{\circ}\right)$, then we shall perform no controlling action.

$\left(1^{\circ}\right)_{2}$ If $H_{1}$ becomes significant in $\left(1^{\circ}\right)$, then we shall perform an action $c_{1}$ such as defined in $\left(1^{\circ}\right)$ in the previous paragraph.

$\left(2^{\circ}\right)$ In either case of $\left(1^{\circ}\right)_{1}$ and $\left(1^{\circ}\right)_{2}$, let us draw a random sample of size $n_{2}$ from the normal population $N\left(m_{1}, \sigma^{2}\right)$ (when $\left(1^{\circ}\right)_{1}$ happens) or from $N\left(m_{1}^{c_{1}}, \sigma^{2}\right)$ (when $\left(1^{\circ}\right)_{2}$ happens), let $\bar{x}_{2}$ be the sample mean defined for $\mathrm{O}_{n_{1}}$, and let us test the hypothesis $H_{2}: m_{2}=\xi$.

Hence we follow the similar procedures as in $\left(1^{\circ}\right)$.

$\left(2^{\circ}\right)_{1}$ If $H_{2}$ becomes non-significant in $\left(2^{\circ}\right)$, then we shall perform no controlling action.

$\left(2^{\circ}\right)_{2}$ If $H_{2}$ becomes significant in $\left(2^{\circ}\right)$, the we shall perform an action $c_{2}$ such as defined in $\left(2^{\circ}\right)$ in the previous paragraph. 
We shall proceed to the third and the following populations in quite similar manners. Thus all the possible forms of distribution functions of the $(k+1)$-th population after these successive $k$ procedures of controls with testing significances will be

$$
N\left(\boldsymbol{m}_{k+1}+\sum_{\nu=1}^{k} \varepsilon_{\nu} f_{\nu}\left(\xi-\bar{x}_{\nu}\right), \sigma^{2}\right),
$$

where $\varepsilon_{\nu}=0,1(\nu:=1,2, \cdots, k)$, and therefore there are $2^{k}$ possible population forms.

There are various types of testing of significances. Since the population variances are equal to a certain known constant $\sigma^{2}$, we may and we shall define the domain of significance in the sample space of $O_{n_{\nu}}$ as $\left|\bar{x}_{\nu}-\xi\right|$ $\geqq A_{\nu} n_{\nu}{ }^{-1 / 2} \sigma$ where $A_{\nu}$ is an assigned positive number.

The effects of introducing testing of significances in control problems will be seen from

Lemma 2.1. Under our general hypothesis in this paragraph, the probability that our second sample $O_{n_{1}}$ will yield its sample mean $\bar{x}_{2}$ which is non-significant in our testing is given by

$$
\begin{aligned}
& \operatorname{Pr}\left\{\left|\bar{x}_{2}-\xi\right| \leqq A_{2} n_{2}^{-1 / 2} \sigma\right\} \\
= & \left\{\Phi\left(A_{1}+n_{1}^{1 / 2} \grave{o}_{1}\right)-\Phi\left(-A_{1}+n_{1}^{1 / 2} \delta_{1}\right)\right\}\left\{\Phi\left(A_{2}+n_{2}^{1 / 2} \delta_{2}\right)\right. \\
& \left.-\Phi\left(-A_{2}+n_{2}^{1 / 2} \grave{o}_{2}\right)\right\} \\
+ & \left.\left\{\int_{-\infty}^{-A_{1}+n_{1}{ }^{1 / 2} \delta_{1}}+\int_{A_{1}+n_{1}{ }^{1 / 2 \delta_{1}}}^{\infty}\right\} \varphi(u)\right\lrcorner\left(u ; A_{1}, A_{2}, \delta_{1}, \grave{o}_{2}, n_{1}, n_{2}, c_{1}\right) d u \\
= & I_{1}+I_{2}, \text { say, }
\end{aligned}
$$

where we put

$$
\begin{aligned}
& \Phi(u) \equiv \int_{-\infty}^{u} \varphi(t) d t \equiv(2 \pi)^{-1 / 2} \int_{-\infty}^{u} \exp \left[-\frac{t^{2}}{2}\right] d t \\
& \grave{o}_{i}=\left(\xi-m_{i}\right) \sigma^{-1} \quad(i=1,2) \\
& \Delta\left(u ; A_{1}, A_{2}, o_{1}, o_{2}, n_{1}, n_{2}, c_{1}\right) \\
= & \Phi\left(\rho\left(\alpha_{2}\right)+n_{2}^{1 / 2}\left\{\left(1-c_{1}\right) \xi \sigma^{-1}+\left(o_{2}-\grave{o}_{1}\right)\right\}+c_{1} n_{2}^{1 / 2} n_{1}^{-1 / 2} u\right) \\
- & \Phi\left(-\rho\left(\alpha_{2}\right)+n_{2}^{1 / 2}\left\{\left(1-c_{1}\right) \xi \sigma^{-1}+\left(\grave{o}_{2}-o_{1}\right)\right\}+c_{1} n_{2}^{1 / 2} n_{1}^{-1 / 2} u\right) .
\end{aligned}
$$

The proof follows from the direct calculation of two parts of the probabjlity of which the first one is the probability of the event $\left|\bar{x}_{2}-\xi\right|$ 
$\leqq A n_{2}^{-1 / 2} \sigma$ under $\left(1^{\circ}\right)_{1}$, while the second one that of the event under $\left(1^{\circ}\right)_{2}$. In fact these two correspond $I_{1}$ and $I_{2}$ in (3.01) respectively. From the standpoint of controls, the following remarks will be useful.

Remark 1. Specially in the limiting case when $n_{1}$ becomes infinite, $I_{1}$ becomes zero and $I_{2}$ tends to the probability

$$
\begin{aligned}
& \Phi\left(\rho\left(\alpha_{2}\right)+n_{2}{ }^{1 / 2}\left\{\left(1-c_{1}\right) \xi \sigma^{-1}+\left(\grave{o}_{2}-\grave{o}_{1}\right)\right\}\right) \\
& -\overline{\mathcal{P}}\left(-\rho\left(\alpha_{2}\right)+n_{2}^{1 / 2}\left\{\left(1-c_{1}\right) \xi \sigma^{-1}+\left(\partial_{2}-\grave{o}_{1}\right)\right\}\right) \text {, }
\end{aligned}
$$

which becomes $\Phi\left(\rho\left(\alpha_{2}\right)\right)-\Phi\left(-\rho\left(\alpha_{2}\right)\right)=\alpha_{2}$ specially when $o_{1}=\delta_{2}$, i.e., $m_{1}=m_{2}$ and $c_{1}=1$, showing that we are abie to attain to the desired standard $\xi$ by letting $n_{1}$ infinite. Neverthless it shows also that gains may not necessarily be secured without the fortunate condition that $m_{1}=m_{2}$ and $c_{1}=1$.

Remark 2. The cost consideration are one of the indispensable standpoints for the control-decision problems. Any gains that would be obtained by making $n_{1}$ larger must be compensated with the costs of making observations of larger amounts.

Theorem 2.2. Under our general hypothesis to this paragraph, the probability density functions $f_{j+1}\left(\bar{x}_{j+1}-m_{j+1}\right)$ of $\bar{x}_{j+1}-m_{j+1}$, where $\bar{x}_{j+1}$ denotes the sample mean of sample $O_{n_{j+1}}(j=1,2, \cdots)$, defined after the successive procedures of statistical controls prepared with testing of significances, satisfy the functional equations in $-\infty<\bar{x}_{j+1}<\infty$,

(3.07) $f_{j+1}\left(\bar{x}_{j+1}-m_{j+1}\right)$

$$
\begin{aligned}
& =f_{j}\left(\bar{x}_{j+1}-m_{j+1}\right) \int_{\xi-A_{j} n_{j}-1 / 2 \sigma-1}^{\xi+A_{j} n_{j}-1 / 2 \sigma-1} f_{j}\left(\bar{x}_{j}-m_{j}\right) d \bar{x}_{j} \\
& +\left(\int_{-\infty}^{\xi-A j n_{j}-1 / 2_{\sigma}-1}+\int_{\xi+A_{j} n_{j}-1 / 2^{2} \sigma^{-1}}^{\infty}\right) f_{j}\left(u-m_{j}\right) f_{j}\left(\bar{x}_{j+1}-m_{j+1}-c_{j}(\xi-u)\right) d u .
\end{aligned}
$$

The proof is immediate from our formulation. It is to be noted that (3.06) yields us the procedures to calculate $\left\{f_{j}\right\}$ recurrently.

\$4. Cost considerations on successive process of regulation with testing of significance. Let us assume that an action $d^{c}$ which will yield us a transformation of population mean $m$ into $m+d$ involves the cost

$$
g(d)=P_{1}|d|^{-1}+P_{2}|d|,
$$


where $P_{1}$ and $P_{2}$ are constants independent of $d$. This is an extremely simplified assumption, which, neverthless, takes into our consideration certain real circumstances. Indeed it may occur very frequently that very small change $|\boldsymbol{d}|$ may involve high costs because of requiring an exact performence, while very large change $|\boldsymbol{d}|$ will mean revolutionary change of apparata.

In the situation when we follow the succssive process defined in Lemma 3.1 , the expected value of the costs of actions will be

$$
\begin{aligned}
\boldsymbol{r}_{c}\left(m_{1}, d_{1}^{c}\right) & =\int_{|\bar{x}-\xi| \geqq A_{1} \sigma n_{1}-1 / 2} \underset{g}{ }\left(c_{1}|\bar{x}-\xi|\right) \varphi\left(n_{1}^{-1 / 2}\left(\bar{x}_{1}-m_{1}\right) \sigma^{-1}\right) n_{1}^{1 / 2} \sigma^{-1} d \bar{x}_{1} \\
& =J_{1}+J_{2}, \quad \text { say, }
\end{aligned}
$$

where, for $\grave{o}_{1}>0$

$$
\begin{aligned}
J_{1}:=P_{1} c_{1}^{-1} \sigma^{-1} n^{1 / 2} \int_{\left|u-n_{1} 1 / 2 \delta\right| \geqq A_{1}}\left|u-n_{1}^{1 / 2} \delta_{1}\right|^{-1} \varphi(u) d u \\
\quad=P_{1} c_{1}^{-1} \sigma^{-1} n_{1}^{1 / 2} \int_{|u| \geqq A_{1}}|u|^{-1} \varphi\left(u+n_{1}^{1 / 2} \delta_{1}\right) d u
\end{aligned}
$$

$$
\begin{aligned}
J_{2}=P_{2} c_{1}\left(\xi-m_{1}\right)\left\{\left(1-\Phi\left(A_{1}-n_{1}^{1 / 2} \grave{o}_{1}\right)\right)-\left(1-\Phi\left(A_{1}+n_{1}^{1 / 2} \grave{o}_{1}\right)\right)\right\} \\
+P_{2} c_{1} n_{1}^{-1 / 2} \sigma\left\{\varphi\left(n_{1}^{1 / 2} \grave{o}_{1}+A_{1}\right)+\varphi\left(n_{1}^{1 / 2} \grave{o}_{1}-A_{1}\right)\right\},
\end{aligned}
$$

where $\varphi, \Phi$ and $\partial_{1}$ are those defined in (3.03) and (3.04) respectively.

We shall readily observe in view of $(4.02)$ and $(4.03)$ that: $\left(1^{\circ}\right)$ for any fixed $A_{1}, J_{1}$ becomes smaller as $\left|\boldsymbol{n}_{1}^{1 / 2} \grave{o}_{1}\right|$ becomes larger; $\left(2^{\circ}\right)$ for any fixed $A_{1}$ and $\xi-m_{1}, J_{1}$ tends to $P_{1} c_{1}^{-1}\left|\xi-m_{1}\right|^{-1}, J_{2}$ to $P_{\varepsilon} c_{1}\left|\xi-m_{1}\right|$ and hence $r_{c}\left(m_{1}, d_{1}^{c}\right)$ to $P_{1} c_{1}^{-1}\left|m_{1}-\xi_{1}\right|^{-1}+P_{2} c_{1}\left|m_{1}-\xi_{1}\right|$, as $n_{1}$ becomes infinity ; ( $\left.3^{\circ}\right)$ for any fixed $n_{1}$ and $\xi-m_{1}, J_{1}$ will become predominat as $A_{1}$ becomes smaller.

\section{Literatures}

Ahrendt, W. R. and Tatlin, J. F.: [1] Automatic feedback controls, 1951.

Deming, W. E.: [1] Some principles of the Shewhart methods of quality control, Mechanical Engineering, 66 (1944); [2] Some theory of sampling, New York (1950); [3] Lectures on statistical controls of quality, Lectures in Japan, (1951).

Dodge, H. F. and Romig, H. G.: [1] A method of sampling inspection, Bell System Tech. J., 8 (1929); [2] Sampling inspection tables; single and double sampling, New York, (1944).

James, H. M., Nichols, N. B. and Philips, R. S.: [1] Theory of servomechanism, New York (1947). 
ShewhaRT, W. A.: [1] Economic control of quality of manufactured products, New York, (1931); [2] Statistical method for the view point of quality contol, Washington, (1939). WALD, A.: [1] Statistical decision functions, New York, (1950).

Wiener, N.: [1] Cybernetics, New York, (1948).

Kitagawa, T.: [1] Successive process of statistical inferences, Mem. Kyushu Univ., Ser. A., 5 (1950); [2] (2) ibid., Ser. A. 6 (1951); [3] (3) ibid., Ser. A. 7 (1952) . 